\title{
Germanica
}

\section{Axel Sandemose}

un outsider persécuté par lui-même

\section{Georges Ueberschlag}

\section{CpenEdition}

Journals

Édition électronique

URL : http://journals.openedition.org/germanica/5287

DOI : 10.4000/germanica.5287

ISSN : 2107-0784

Éditeur

Université de Lille

Édition imprimée

Date de publication : 30 juin 1987

Pagination : 173-208

ISSN : 0984-2632

Référence électronique

Georges Ueberschlag, «Axel Sandemose », Germanica [En ligne], 1 | 1987, mis en ligne le 05 octobre 2018, consulté le 06 octobre 2020. URL : http://journals.openedition.org/germanica/5287 ; DOI : https://doi.org/10.4000/germanica.5287

Ce document a été généré automatiquement le 6 octobre 2020.

(c) Tous droits réservés 


\section{Axel Sandemose}

un outsider persécuté par lui-même

\section{Georges Ueberschlag}

1 Pourquoi un écrivain se sent-il né sous une mauvaise étoile - telle est la question obsédante qu'Aksel Sandemose n'a cessé de se poser tout au long de sa vie. Il se met enquête d'explications, accuse, sonde son passé et son propre moi, s'insurge. Perpétuellement en fuite, se fuyant lui-même et fuyant le monde étriqué de ses origines, tel un marin qui a jeté des bagages par-dessus bord, ce "Strindberg norvégien » comme on l'a appelé, se sentira un étranger en révolte, un malchanceux en rupture de ban, s'obstinera à défaire un monde bête et injuste qu'il poursuivra d'une haine tenace.

2 Nourrie de son expérience personnelle, cette haine deviendra peu à peu la mesure de toute chose. "Finalement je ne connais qu'un seul homme, un homme et sa haine", confiera-t-il un jour au critique et écrivain Sigurd Hoel ${ }^{1}$. Son combat, il le mènera donc avec la rage folle de ces anciens guerriers scandinaves, les Berserker, dont la croyance populaire nordique disait qu'ils étaient capables de prendre la forme d'un ours et de se battre les mains nues, animés d'une force démentielle.

3 Scandinave, Aksel Sandemose l'est totalement, par ses origines et par son destin. Né en 1899 dans la petite ville danoise de Nykøbing sur l'île de Mors d'un père danois et d'une mère norvégienne, il a vécu tour à tour au Danemark, en Norvège et en Suède, tout en sillonnant les mers arctiques dès son jeune âge.

4 Scandinave, il l'est aussi par son œuvre, une longue et subtile variation sur un même thème, celui du banni, du fugitif qui revient sur ses pas.

5 La famille d'Aksel Sandemose était d'origine modeste. Son grand-père, vétéran de la guerre des Duchés; où il avait perdu sa jambe gauche, était un esprit vagabond qui vivait d'expédients et de mendicité. C'était un de ces personnages de folklore local qui nourrissent l'imagination des campagnes. Il sombrait volontiers dans l'alcoolisme, tout comme ses nombreux enfants humiliés par cette misère.

6 Mais le père d'Aksel fit exception. Il se révolta contre la fatalité de l'alcool et s'enfuit de la maison paternelle. En signe de protestation et par réaction, il devint un fanatique 
militant anti-alcoolique. Ce fut un homme de principes, ouvrier consciencieux, fier de sa morale du travail, un rocher pour ses enfants. Il incarna un monde de traditions et d'ordre que l'écrivain, son fils, allait soupçonner en bloc. Sa mère, d'origine norvégienne, avait su garder un certain mystère autour de sa personne. Sa peur étrange de la mer semblait provenir de quelques années passées comme émigrée dans les mers du sud. Avec ses neuf enfants, elle eut amplement l'occasion de pratiquer le sens de l'économie et l'héroïsme du quotidien.

7 La famille vivait à l'unisson de la population danoise de l'île de Morsø. Ouvriers, paysans ou marins, ils étaient tous habitués à lutter pour leur survie dans la grisaille de tous les jours, surpris et heureux parfois de voir qu'un beau jour de juin pouvait transformer leur petite île en idylle au soleil.

\section{Les débuts d'une révolte}

Dès l'âge de quatre ans, le jeune Aksel essaya de mettre le feu à la maison paternelle. Cet acte irraisonné, instinctif, fut comme une préfiguration de sa vocation future d'écrivain pyromane, démasquant les hypocrisies du monde et brûlant leurs demeures. Comme il l'a voulu faire de la petite masure familiale, il poursuivra plus tard de ses foudres la ville tout entière, appelant sur elle les feux de la destruction, le feu purificateur.

9 Jante, nom qu'il donnera dans ses œuvres à sa ville natale, sera le symbole de la tyrannie collective qui s'enroule dans ses préjugés comme dans un cocon, qui règne par la vertu toute puissante du "normal », où l'individu est incapable de se défendre. Objet de son mépris, il voudrait la voir réduite en cendres, tout en ne pouvant s'empêcher de pleurer secrètement sur la cité perdue.

10 À l'école, le jeune Aksel promène sa mine triste et peureuse. Il craint l'Autorité et tous ceux qui l'incarnent, mais il apprend avec facilité. Premier de la classe, il ne peut cependant rien contre le fait qu'il appartient au clan des «B», des enfants de prolétaires. La rage au cœur, il se voit obligé de suivre ceux de ce clan, sans possibilité de choix, lorsqu'ils livrent parfois une véritable guerre aux autres, aux « $\mathrm{A}$ », aux enfants des bourgeois.

11 L'école a contribué à faire de l'enfance d'Aksel Sandemose une longue période d'humiliations. Dans cette ambiance de lutte des classes, au sens exact du terme, les inégalités sociales ne sont cependant encore contestées que par quelques petits frondeurs.

12 Sandemose lira très jeune, dans le journal social-démocrate local, Pelle le conquérant de Martin Andersen Nexø. Mais il ne partagera jamais l'idéalisme optimiste de Nexjø qui considère les pauvres comme des élus appelés à la Terre promise. Un autre scandinave, Strindberg, le fascine bien davantage. Comme lui, il se sent « fils de la servante », blessé dans son sens de la justice. Même égocentrisme chez l'un et chez l'autre, même besoin de révolte et même sentiment obscur de culpabilité.

Dès l'âge de quatorze ans, année de sa confirmation et de son entrée dans le monde des adultes, Sandemose se croit marqué par un sombre destin, condamné à appartenir au Mal. Il veut masquer son sentiment d'infériorité qui en résulte par un comportement fanfaron et orgueilleux. 
14 Et c'est ainsi, par besoin de revanche, qu'il s'oppose à son père. Malgré son admiration pour lui il refuse son autorité et sa morale de la tempérance. Initié par ses semblables à la religion de l'eau-de-vie, il se met à boire. L'alcool devient l'arme de la révolte sociale, la première, sous le signe de la misère et de l'ignorance. Les pauvres et les adolescents en révolte ont ainsi leur façon à eux de proclamer les droits de l'homme. Pour prouver sa virilité on boit et on se saoule. Sus à la tyrannie des conventions, à celle du père et de son idéal de tempérance !

15 L'expérience, l'habitude, le besoin incoercible de l'alcool se profileront sur toute l'œuvre de Sandemose. Est-ce une maladie de l'âme due à la peur, au complexe d'infériorité, à la solitude? Quoiqu'il en soit, les Ligues de tempérance sont de mauvais médecins pour Sandemose.

16 Comme tant d'autres écrivains issus des milieux prolétariens, Sandemose profite de la tradition scandinave d'éducation populaire qui ouvre le Parnasse aux fils du peuple. Au cours de son séjour dans une højskole il découvre son attrait pour la langue, arme au moins aussi redoutable que l'alcool. Décrochant un vague diplôme d'instituteur suppléant, il se retrouve à Nykøbing, en proie à ses conflits de conscience, à ses tiraillements et à la pression de son entourage. Pour y échapper il s'engage en 1916 comme matelot sur un petit navire suédois.

17 Ce ne sont ni l'appel de la mer ni le romantisme de l'aventure qui le poussent, il le fait presque sur un coup de tête, pour s'échapper. Et le voilà donc sur ce caboteur suédois, véritable cercueil flottant, sur lequel s'agitent quelques marins soudards et bagarreurs qui tyrannisent le jeune mousse. Une tyrannie de plus!

18 Celui-ci tient son journal, y décortiquant déjà les mécanismes et les raisons psychologiques profondes de ce qui lui arrive. Le ton en est détaché, mais la blessure d'amour-propre est profonde. Elle jettera sa longue et sinistre ombre sur l'œuvre ultérieure.

19 Malgré la discipline que le capitaine impose sur le bateau au cours de la traversée pour Terre-Neuve, le jeune mousse ne se sent pas à l'abri des autres. Le dégoût pour la vie de marin lui vient rapidement. Le 23 octobre 1916 Sandemose déserte le bateau et s'enfuit à la nage. Au bout de quelques jours il se retrouve embauché dans un camp de travail, en pleine forêt arctique, au milieu d'autres brutes analphabètes. Le tout est d'une tristesse infinie, et il continue à tenir son journal. Cette fuite du 23 octobre deviendra une des scènes centrales ouvrant l'univers romanesque et mythique de Sandemose.

Revenu au pays sur un autre bateau, il retrouve la même impression d'étroitesse, de stagnation, de désespérante monotonie. Jante restera toujours Jante. Rien n'y arrive, sauf le rêve qui vous tourmente et qui est la serre où mûrit la vocation d'écrivain.

21 Aksel Sandemose part donc pour Copenhague où il suit des cours du soir et continue à se cultiver en autodidacte. Il fait l'expérience de l'amitié et de la poésie. Plein de dédain pour les écrivains engagés, il se veut déjà au-dessus de la mêlée sociale. Il admire Baudelaire et Verlaine, et se sent des affinités avec le poète norvégien Sigbjøn Obstfelder ${ }^{2}$. De vastes projets enthousiastes s'échafaudent déjà, des romans à écrire, tous autour du même thème : le conflit de l'individu avec son entourage, la haine de la société et la psychologie de celui dont elle a fait un criminel. Mais ces projets se heurtent à la pénurie de l'inspiration, et les manuscrits de Sandemose seront refusés à plusieurs reprises par Gyldendal. 
En 1921 cependant paraît dans le Morsø Folkeblad son premier petit écrit en prose, Hjemløse Fugle (Oiseaux sans patrie), où il décrit son retour amer à Nykøbing. La même année il se marie avec une amie d'enfance, et pour subvenir aux besoins de la famille il accepte toutes sortes de travaux, essaie de vendre des livres d'édification religieuse. Mais il continue rageusement d'écrire en cachette. La discipline de la vie, le travail régulier, l'intégration sociale, il ne peut décidément pas s'y soumettre, et il lui semble avoir hérité des romantiques une horreur tenace du philistin.

Par chance, Sandemose fait la connaissance, en 1923, de l'écrivain danois Johannes V. Jensen. Celui-ci, par amitié pour ce jeune ambitieux, le tire de son anonymat et de sa désespérance en publiant quelques-uns de ses récits dans la revue Forum qu'il venait de lancer. Jensen lui conseille de travailler sa technique et son style à l'école des anglosaxons. Il croit en ce génie naissant, et c'est sur sa recommandation que Gyldendal accepte la même année de publier les Fortaellinger fra Labrador (Récits du Labrador).

Tout comme Jensen avait déjà tenté de capter l'écho des vieilles sagas islandaises, Sandemose essaie dans ces nouvelles de recréer l'atmosphère mythique des temps scandinaves anciens.

Sur fond de paysage rude et hostile se déroulent des drames sauvages où les lois de la sélection naturelle et de la lutte pour l'existence priment tout. Des amours rudes et contrariées s'y mêlent, et la jalousie fait ses ravages dans des drames triangulaires. La femme y joue souvent un rôle accessoire, c'est l'enjeu, simple décor même, que le vainqueur qui a éliminé son rival laisse volontiers en pleurs au bord du chemin, en s'en allant d'un air indifférent.

Sandemose plonge son lecteur dans un univers de la force nue et du vent glacial où les sentiments sont un luxe superflu. Le lynx y arrache les entrailles des jeunes de la louve, en attendant que la meute le rattrape et le déchire à son tour. La vie, ce sont des taches sanglantes sur la neige infinie, ce sont des marins qui luttent désespérément contre les éléments avant que l'océan ne les engloutisse, des marins qui mettent leur virilité et leur force en compétition jusqu'à complet épuisement. L'honneur du mâle !

L'âpreté des anciennes sagas se retrouve presque à chaque page de ces nouvelles. On pourrait penser qu'elles font de Sandemose aussi un poète de la mer. Mais celle-ci ne fournit que le cadre du drame central, celui de la libération de l'individu par sa propre force. C'est le premier thème majeur de l'œuvre de Sandemose, autour duquel s'ordonnera toute sa production de jeunesse. Fuir le troupeau des faibles et la terreur du collectivisme, devenir un héros, un chanceux, un battant qui se lance dans la conquête optimiste du monde. propre mythe, dont les deux pôles seront le guignard et le chanceux - les deux faces de son autoportrait, la réalité et l'idéal. Il sent comme l'une le tenaille, comme l'autre le séduit. C'est ainsi qu'il se met lui-même en scène dans deux romans, Maend fra Atlanten (Hommes de l'Atlantique) et Ungdomssynd (Péché de jeunesse), s'incarnant dans deux personnages dont l'un triomphe en retournant dans sa ville natale, et dont l'autre se suicide.

Dans les années 1920-1930 le chanceux, 1'optimiste conquérant de l'avenir sera le personnage central chez Sandemose. Se sentant ballotté entre son désir de communion et son besoin d'indépendance, il revit la problématique ambition déjà décrite, quoique 
sous un autre éclairage, par Henrik Pontoppidan dans Lykke Per (Pierre le chanceux). Pour échapper à la prison de la société, il n'y a que la solitude et le culte du surhomme.

Le premier à réaliser ce rêve audacieux dans l'œuvre de Sandemose, c'est l'intrépide Erik Skjelholm, le héros de la nouvelle Manden fra Hulen (L'Homme de Hulen). Il refuse de se plier aux conventions, se révolte contre la dictature du nivellement et découvre la pleine liberté sur une île déserte. La protestation anarchisante qui s'élève de Manden fra Hulen ne s'éteindra plus chez Sandemose. Mais il la placera de préférence dans la bouche de prophètes sombres et malheureux, comme ce Kresten Ravnborg, personnage principal de la grande nouvelle I livets korridor (Dans le couloir de la vie). L'action de cette nouvelle se passe dans le milieu d'une École Supérieure Populaire, et son héros, Ravnborg, est déjà une première esquisse d'Espen Arnakke.

Les succès de librairie que Sandemose avait connus sous la houlette de Jensen semblent cependant sans lendemain. Il rencontre des difficultés économiques, doit occuper pendant deux ans un poste de gardien à la Glyptothèque de Copenhague. En 1927, il part au Canada comme correspondant du journal Berlingske Tidende, et il réussit, après plusieurs refus, à placer le manuscrit de son premier grand roman de la mer, Klabautermanden.

Le personnage en est une figure mythique, comme le Hollandais volant. Mais alors que ce dernier garde la fierté de l'ange déchu, Klabautermanden est un ange du malheur qui entraîne dans le naufrage tous les marins qu'il rencontre dans son errance sur l'océan. Son histoire est celle d'un homme vaincu par ses instincts érotiques, dans sa lutte contre la femme qui se refuse. Ame damnée, sans rédemption, il est obligé d'errer éternellement sur la mer.

Klatautermanden, roman de la chute d'un orgueilleux qui se croyait un surhomme, prépare Sandemose psychologiquement à reconnaître qu'il n'est pas le chanceux qu'il espérait être, mais celui que la guigne a marqué de son empreinte.

Cet aveu, pénible et libérateur, amorce déjà la grande confession d'Espen Arnakke dans En flyktning krysser sitt spor (Un fugitif revient sur ses pas).

Ne trouvant aucune satisfaction profonde dans sa vie au Canada, où il fréquente quotidiennement des émigrés danois de l'Alberta qui végètent et cultivent leur nostalgie du pays, Sandemose décide de retourner en Europe, avec l'idée de s'établir en Norvège, pays des légendes et pays de sa mère. Incité par l'exemple de l'écrivain Jørgen Bukdahl qu'il avait connu chez J.V. Jensen, et qui s'était déjà établi en Norvège dès 1925, il part pour Oslo au mois de mai 1930, emportant le manuscrit d'un nouveau roman, En sjømann går iland (Un marin retourne à terre).

Aucune émotion, aucune larme n'entourent cet exil-retour, qui ressemble à une nouvelle fuite. Mais celle-ci n'évitera pas au garçon de Jante la grande explication avec son passé. Le changement de nationalité ne pouvait masquer sa défaite qu'en apparence. Si ses rêves s'étaient effondrés au contact de la réalité, s'il ne pouvait être le chanceux, il y avait des motifs. Il fallait donc les démasquer. Le grand règlement de compte avec Jante n'allait que commencer.

\section{Les arcanes d'une histoire}

Aksel Sandemose avait mal choisi le moment de son exil-retour. Il arrive à Oslo alors que la grande dépression économique atteint la Norvège de plein fouet. Le 
gouvernement de droite y doit faire face à des difficultés et à une hostilité croissantes. Le mouvement d'inspiration marxiste « Mot Dag » mène la lutte sous la direction d'un fanatique froid et résolu, Erling Falk.

Parmi les intellectuels engagés dans les rangs de «Mot Dag » se trouve l'écrivain Johan Vogt. Sandemose fait rapidement sa connaissance à Oslo. Tout en restant dans la sphère d'intérêt et la mouvance de ce dernier, il gardera ses distances à l'égard de " Mot Dag ", tel un satellite lointain. Car il a plus urgent à faire que de se lancer dans l'action politique. Pour donner plus librement cours à sa révolte contre Jante, il change de langue et traduit le manuscrit de En sjøman går $i$ land en norvégien. Dorénavant, c'est dans cette langue qu'il écrira.

Ce roman raconte l'histoire d'un jeune marin, Espen Arnakke, qui déserte le bateau, comme le fit déjà Sandemose un certain 23 octobre. Arrivé à terre, il trouve du travail parmi les bûcherons de Misery Harbor. Un de ses camarades de travail, John Wakefield, beau et bien musclé, l'accueille avec sympathie, car il trouve "quelque chose de féminin » dans la façon d'être et de se comporter du nouveau venu. Mais voici qu'arrive Eva, la belle Eva. Encouragé par ses tentatives antérieures auprès de quelques laiderons, Espen Opse lever les yeux vers elle, car il se rend compte que c'est par les filles que l'homme atteint pleinement son assurance et son autorité.

Eva ne semble pas rester insensible, et Espen se voit déjà... le rêve court, et Jante en restera bouche bée lorsqu'il leur montrera quel homme il a su devenir !

Mais voilà, John, l'irrésistible John le supplante facilement. Rien n'a donc changé, et les expériences malheureuses qui ont conduit Espen d'humiliation en humiliation se poursuivent ici. Il est même obligé d'écouter, impuissant, les ébats amoureux de John et d'Eva.

42 Sa première pensée est de cacher à tous cette nouvelle défaite, et il boit, vidant la bouteille d'eau-de-vie avec laquelle il avait voulu acheter l'amitié de John. Mais lorsque celui-ci le raille et le ridiculise, en lui révélant qu'il connaît son inutile passion pour Eva, en voulant l'entraîner même à singer avec lui les embrassades, tout s'effondre. Et Espen frappe, frappe avec son couteau sous l'empire de la jalousie et de l'ivresse. C'est comme s'il sortait de ses gonds, et lorsque le corps de John n'est plus que «quelque chose qui gît là » et qu'il regarde de loin, il se dit avec un calme froid: Te voilà donc devenu un assassin, Espen Arnakke !

La rencontre avec l'incomparable, le puissant John Wakefield, dont la figure et le nom même font penser à J.V. Jensen, l'a replongé dans l'éternelle constellation du chanceux et du guignard. Il est devenu l'assassin de son rival à Misery Harbor. Sans le vouloir vraiment, sans en avoir nettement conscience, il a tenté de se défaire par le meurtre de l'inextricable filet dont l'entoure sa jalousie, son admiration haineuse pour John.

Misery Harbor est l'endroit quasiment mythique dans l'univers romanesque de Sandemose, où Espen Arnakke a touché le fond de son avilissement, mais où commence également à poindre l'aube du salut et de la délivrance. $O$ felix culpa !

Le marin qui est retourné à terre est devenu un meurtrier, mais il devra encore attendre avant de revenir sur ses propres pas et de reconnaître le chemin tortueux qui l'a mené de son enfance jusqu'au lieu du drame.

Pour l'instant il tente encore de masquer, d'occulter les raisons et les motivations profondes de son acte, en essayant de jouer les matamores dans les cabarets de la 
prairie canadienne où il s'est sauvé, de courir après l'image de John, surhomme irrésistible, qui représente tout ce qu'il avait voulu être lui-même.

À bout de souffle, voyant l'inanité de ses efforts, il doit reconnaitre qu'il reste le prisonnier de son passé et de sa déveine. Même au Canada, Jante ne le lâche pas.

Alors même qu'il vient de publier ce roman, Sandemose pense trouver enfin une clé qui lui permettra d'ouvrir son monde intérieur, de rebrousser chemin et de revenir sur les pas de son enfance. Il commence à s'intéresser au freudisme qui jusqu'à présent ne l'avait guère encore attirés. Il s'en était même tenu volontairement éloigné, à cause de son admiration pour J.V. Jensen qui avait jeté l'anathème sur la psychanalyse et l'introspection. Il se rend ainsi compte que l'acte libératoire de Misery Harbor a rendu Espen disponible, capable de vaincre ses inhibitions, de faire taire « les voix intérieures qui censurent».

Espen Arnakke - Aksel Sandemose, la similitude entre les deux personnes n'a plus besoin d'être soulignée.

50 Et Sandemose commence à écrire dorénavant à la première personne. Le «je » fait surface, et le moine fait plus trembler. La méthode psychanalytique de Freud devient le rituel de l'exorciste. On chasse le démon, fût-ce, pour Sandemose, sous le signe du rationalisme.

51 Sigurd Hoe ! avait engagé Sandemose, à la suite de la publication de En sjømann går iland, à poursuivre le récit de cette libération. Sandemose lui répondit, et cette correspondance déclencha en lui un mécanisme de relaxation. Sa lettre, dont le livre gardera des traces et des réminiscences, le destinataire y étant souvent interpellé directement, prendra des proportions insoupçonnées et deviendra un manuscrit volumineux de plus de mille pages. En flyktning krysser sitt spor fut écrit en quelques mois, comme en transes. Sandemose avait trouvé l'enveloppe matérielle de sa propre histoire.

52 Ce livre, document impitoyable d'une crise personnelle, commence par la phrase: "Maintenant je vais tout raconter ». Ces mots sonnent comme le cliquetis de chaines qui tombent enfin. Le mécanisme psychique se débloque, et la grande confession de l'auteur s'étalera de page en page. Une confession qui sera en même temps un appel à la révolte et le roman du devenir d'un homme.

53 Les mots semblent se précipiter, les associations d'idées se bousculent, les sentiments étouffés et les inhibitions réclament leur droit à la parole. Le malade étale dans un monologue inlassable sa vie trouble. Le flot incohérent des souvenirs s'écoule, tandis qu'un virtuose de la maïeutique, caché à l'intérieur même du confessant, semble veiller au bon déroulement de l'accouchement. Cette gigantesque auto-analyse fut rédigée dans un pays en pleine crise, à une époque de guerre idéologique où la menace des dictatures se précisait. Mais le livre de Sandemose parlait d'une autre dictature. C'était comme une éruption volcanique, et le volcan était situé dans un univers autre, où l'actualité n'avait pas de prise.

54 Sigurd Hoel, qui lut le manuscrit, proposa à Sandemose de le couler « dans un langage civilisé » afin de le publier. "Ce sera alors un roman unique en son genre », ajouta-t-il. Sandemose refit le manuscrit et l'envoya en 1932 à Gyldendal. Sous prétexte que «ce n'était pas ce qu'on appelle un livre », craignant en réalité les critiques de l'opinion et la réaction des conservateurs, la maison d'édition le refusa ${ }^{4}$. On vivait alors en Norvège dans une atmosphère de chasse aux «kulturradikala », et l'on pouvait craindre que le 
roman de Sandemose ne fût ressenti comme un long réquisitoire contre les conventions et contre la morale.

En flyktning krysser sitt spor (Un fugitif revient sur ses pas) ${ }^{5}$ fut cependant publié en 1933 par Tiden Norsk Forlag, la maison d'édition que le parti ouvrier norvégien venait de créer. Le livre sera bientôt reconnu par la critique comme une des grandes œuvres de la littérature scandinave des années 1930-1940. Sandemose le reprendra en 1955, et c'est cette édition, remaniée, que nous analyserons plus loin plus en détails.

Le livre, choquant et provocant par ses accents inédits, ses passions, ses haines, sa forme chaotique, s'attaquant à des sujets tabous, dégageant une atmosphère morbide certaine, déclencha les attaques des réactionnaires d'Oslo. Mais Sandemose, qui savait que c'est justement dans les petites capitales que les hommes peuvent le plus facilement cuire dans leur propre jus, fit résolument front. Il annonça la publication d'une nouvelle revue, Fesja ${ }^{6}$, dont il serait le seul rédacteur, et la parution dans cette revue d'une postface à son roman. Persistant et signant, il intitula cette postface, avec une ironie évidente, L'apôtre de la vertu.

57 Le succès du roman dans les pays voisins, sa traduction en Amérique, avec une préface de Sigrid Undset, le confirmèrent dans son attitude de provocation. Défiant la société, il voulait montrer par son propre exemple comment celle-ci organisait la dictature sur les esprits. Il contribua même à créer sa propre légende en Norvège, nourrissant les potins et les rumeurs qui couraient au sujet des bacchanales dont il aurait été le centre au Café du Théâtre à Oslo.

58 En 1935 Sandemose publia, dans la foulée, un nouveau roman scandaleux, Vi pynter oss med horn (Matelot de Norvège) ${ }^{7}$. L'alcool et le sexe forment les deux axes de ce roman. L'un règne sans retenue - «l'alcoolisme, c'est l'évasion hors du malheur », proclame l'auteur dans le premier des commentaires didactiques dont il accompagne les chapitres. L'autre se cache derrière un étrange voile de pudiques non-dits, malgré la constante présence d'instincts sadiques. L'amour chez Sandemose, dans presque toutes ses œuvres, est d'ailleurs étrangement dépourvu de chaleur, malgré les éruptions passionnelles. Les scènes érotiques restent froides, comme si la plume de l'auteur était inhibée par la peur du corps, la peur de la femme.

Vi pynter oss med horn est un $\mathrm{d}$ ame de l'amour et de la jalousie. Dès sa publication, le livre fut attaqué par le président du Storting, le parlement norvégien, comme étant "de la littérature dégoûtante». Il raconte le conflit qui oppose deux matelots du Fulton, un vieux brick, durant une traversée en Mer du Nord. L'ambiance malsaine. et la tension qui règnent sur le bateau et qui laissent au lecteur une impression de profond malaise, ne se relâchent jamais. Durant six mois tous les instincts sexuels se libèrent en beuveries, rixes et lubies sadiques.

60 L'un des matelots, Prasten, de son vrai nom Johannes Hansen, est une nature introvertie. La configuration psychique est manifestement très voisine de celle d'Espen Arnakke. Depuis son enfance il a été l'objet de sarcasmes, et il s'est réfugié dans des études de théologie. Mais à cause du viol d'une jeune fille il a été obligé de fuir et de s'engager comme matelot.

61 Sur le bateau il se voit rapidement exposé aux vexations de l'équipage. Il devient en particulier le souffre-douleur de Gullhästen, son parfait antagoniste, qui l'oblige à subir ses vexations et ses violences. Ivrogne et brutal, le type même du bellâtre rustre, celuici règne en despote sur l'équipage. Mammouth solitaire, il en est le « chef ». Prästen lui 
voue une secrète admiration, car il est le chanceux. Mais en même temps il sent grandir sa haine. Gullhästen, en mâle despote, lui interdit de s'approcher de Helga, la superbe Islandaise, ne serait-ce que parle biais de l'image qu'il en a sculptée, celle de la déesse Gulnare.

Cette image de l'inaccessible symbolise assez bien la conception de la femme chez Sandemose. À la fois madone et putain, elle appartient aux rêves de tous, mais humilie celui qui se donne à elle. Elle reste confinée dans le domaine du sexe. Certes, Sandemose s'est prononcé pour l'émancipation de la femme, sur le plan des principes il ne pouvait guère faire autrement. Mais ses héros romanesques restent, tout comme luimême, prisonniers d'une conception machiste et réactionnaire. Gullhästen, Prästen et Helga se meuvent dans cette relation triangulaire où la jalousie et la haine amoureuse du rival heureux, aux relents homosexuels ${ }^{8}$, servent de moteur. La jalousie traverse d'ailleurs toute l'œuvre de Sandemose comme un loup-garou. La jalousie fait partie de la constellation érotique, elle use les sentiments jusqu'à les rendre exsangues.

Prästen, comme Espen, comme plus tard John Torson ou Erling Vik, est celui qui arrive toujours en troisième position, lorsque le rival est déjà là. Élevés dans l'étroitesse qui les incite à la haine, ces "héros" cherchent leur revanche auprès de femmes inaccessibles et forment avec leur rival le constant duo du chanceux et du guignard. L'ombre de John Wakefield les poursuit tous.

La publication de ce roman, dont Johan Bargen fut un des premiers à souligner la valeur, correspond chez Sandemose à la montée de nouvelles années de crise. Crise personnelle d'abord, qui a bout ira à son divorce, mais aussi crise européenne due à l'arrivée au pouvoir du nazisme.

Dès 1934, dans un long essai intitulé Dødens Agenter (Les Agents de la Mort) Sandemose met le monde en garde contre la course aux armements. Dans le journal socialiste "Arbeiderbladet » il s'élève dans des articles répétés contre le danger du nazisme, ses adeptes zélateurs comme Vidkun Quisling, et contre toute tentative de minimiser le mal. Mais il reste sans illusion sur la portée pratique du pacifisme idéaliste et pense que la paix ne peut être garantie que si elle répond à des intérêts économiques.

Cet engagement par la plume a donné lieu, rétrospectivement, à une interprétation beaucoup plus politique de Vi pynter oss med horn. Partant du postulat que le brick Fulton représente la société capitaliste et son équipage la classe ouvrière, Ulla Alfredsson ${ }^{9}$ voit dans ce roman une tentative pour expliquer la montée du fascisme par l'approche de la psychologie des foules. Sandemose essaierait de montrer comment la conscience de la classe ouvrière est anéantie par une sexualité perverse et un langage faussé, ce qui expliquerait pourquoi, se rapprochant de la petite bourgeoisie, elle peut devenir un soutien accidentel du fascisme.

Cet essai d'explication, qui doit beaucoup au freudo-marxiste Wilhelm Reich, est, certes, intéressant, mais néglige complètement le fait que les deux facteurs de la sexualité et de la langue portenteneux, pour Sandemose aussi, un potentiel d'émancipation. Quoiqu'il en soit, Sandemose renonce rapidement à tenir le devant de la scène politique, s'en écarte même délibérément, poussé par ses démons intérieurs qui le ramènent toujours aux mêmes problèmes personnels, aux mêmes obsessions.

Det stod en benk i haven (Un banc dans un jardin), roman écrit à la hâte, retrace, encore, la vie du jeune Aksel - Espen à Nykøbing, analyse ses complexes d'infériorité. Lorsque le jeune garçon de dix ans refuse de donner la main au roi qui vient rendre visite à ses 
sujets de Jante, ce n'est pas par esprit de rébellion, mais parce que l'autorité le paralyse. lorsqu'il tente, plus tard, de faire ses preuves en buvant, malgré l'interdiction paternelle de toucher à la bouteille diabolique, et en courtisant les filles, il récolte échec sur échec. Ce drame de la puberté sera repris et intégré dans la version remaniée de 1955 du livre-clef de Sandemose En flyktning krysser sitt spor.

Pour le moment Sandemose voyage. Il séjourne en Terre-Neuve, puis aux Indes, envoie des articles au journal Politiken. C'est comme un pèlerinage sur les traces d'Espen. Sandemose le raconte dans Brudulje. Lorsque son train s'approche de Misery Harbor, l'endroit fatidique, Espen est paralysé de peur, s'attendant à être terrassé par la Némésis. Mais rien n'arrive. Misery Harbor n'est qu'un petit coin perdu en marge de la vie.

La volonté de survivre arrache Espen à son passé, mais non pas à l'univers clos de ses cauchemars et de ses fantasmes. Sandemose adhère tellement à ces thèmes majeurs de la perversion de l'éducation, de la dictature de la masse et de la jalousie du "troisième", à ces éléments avec lesquels il construit, patiemment, son mythe personnel, qu'on les retrouve même dans le roman September qui forme avec Ross Dane (titre norvégien Nybyggere $i$ Alberta - Colons de l'Alberta) et En sjømann går iland la trilogie où l'auteur raconte son expérience des émigrants danois de 1'Alberta.

\section{Une saison en enfer}

71 De retour en Norvège en 1940, alors que la guerre fait déjà rage, Sandemose publie, comme pour détourner ses regards de la tempête, un recueil de récits, Fortellinger fra andre tiden (Récits d'un autre âge). Ce sont des petites nouvelles, des anecdotes, qui ne représentent qu'un moment de répit dans la grande crise qui approche de son apogée.

La Norvège est occupée par les nazis. Sandemose, qui se place tout naturellement du côté de la résistance, mais en gardant prudemment ses distances, y voit la preuve de la crise morale, la confirmation de la décadence, et éprouve le sentiment d'être parmi les responsables. Recherché par la Gestapo, ou croyant l'être, il s'enfuit en Suède. Mais à Stockholm, malgré l'amitié d'Eyvind Johnson, il est mal reçu, comme presque tous les réfugiés norvégiens. Il lui arrive de nouveau de se comparer à Rimbaud et de penser qu'il passe sa saison en enfer. Les Suédois, égoïstement penchés sur leur propre confort, montrent une incompréhension profonde, accumulent les vexations contre les réfugiés. Jante, le royaume de la bêtise, se trouve décidément partout. Sandemose vit dans une atmosphère de tension psychologique extrême. Il s'adonne de plus en plus à l'alcool, devient agressif, s'aliène des amis, même dans le milieu des réfugiés norvégiens, et il va jusqu'à gifler publiquement Ivar Lo-Johansson. Il se sent étranger au monde qui l'entoure, incompris de lui. La solitude commence à se faire plus pesante, plus nécessaire parfois.

73 Elle est le thème central d'un nouveau roman, Tjaerehandleren (Le marchand de goudron), qui raconte l'histoire d'un criminel. Audrun Hamre, dont les initiales sont les mêmes que celles d'Adolf Hitler, est un solitaire paranoïaque. Fils naturel d'une servante de ferme, il poursuit son père d'une haine implacable. Ayant émigré aux États-Unis, il y a plus de trente ans, il revient en Norvège à la veille de la guerre. Là, il se trouve mêlé à une obscure affaire criminelle, et il s'avère qu'il en est à la fois le détective et le meurtrier. Son mariage avec une femme volage n'a été qu'un leurre, mais il l'a poussé à fréquenter le monde des sectes. Des prédicateurs cyniques lui ont appris comment 
appeler et dominer les démons de l'hystérie collective et faire des affaires sur le compte des âmes blessées. Son crime n'a pas de complices, il se cache même derrière les apparences de la morale et du puritanisme.

Avec une lucidité sans ménagements, Sandemose analyse dans cette œuvre la psychologie du «führer », mais aussi celle des masses anonymes qui se laissent mener par ceux qui ont le pouvoir. La démocratie en de vient une vaste illusion collective, une cynique escroquerie à l'image de Jante.

C'est dans la même ambiance pesante de son exil suédois que Sandemose rédige un autre roman sur fond autobiographique, Det svundne er en drøm (Le Passé est un rêve). Il y reprend des thèmes et des éléments de l'œuvre précédente.

76 En fait, il s'agit moins d'un roman que d'un journal collant à l'actualité, celle de la guerre. Sandemose voulait d'abord y décrire la Norvège vue par un émigré qui revient au pays. Mais les années d'occupation ont changé la finalité du livre, et la résistance norvégienne contre l'occupant y prend une place plus importante.

Se faisant moralisateur, l'auteur veut donner une nouvelle perspective au sens de la vie, expliquer les catastrophes collectives. Le destin de l'homme lui apparaît comme l'image du destin du monde, ou inversement, les règles éthiques étant les mêmes pour les deux.

78 Le retour en Norvège de John Torson, qui a émigré en Amérique pour des raisons assez obscures, correspond donc aussi à un retour sur lui-même. En véritable détective de la conscience humaine, un peu dans l'esprit proustien, il revient sur ses pas, explore le passé à l'endroit même où son frère, son alter ego, un jour a abattu son rival. Par le fait des circonstances et de l'actualité il devient lui-même, une nouvelle fois, un meurtrier, et il se plonge dans les arcanes, les tenants et les aboutissants de ses actes.

79 Sandemose est fasciné par le crime de ses héros, tout tourne autour de ce crime, comme le cyclone tourne autour de son œil. Le crime d'Espen Arnakke, qui a poignardé son rival et bourreau John Wakefield, avait été mis en relation avec une situation de terreur sociale. Ici, en revanche, Sandemose part à la recherche des ramifications psychologiques inconscientes du crime. Il a lu E.A. Poe, ses analyses de l'âme humaine, de ses motivations et de ses actes irrationnels, et il met l'accent sur les tendances auto destructrices qu'il y décèle. La vision romantique d'une vie psychique démoniaque, à la E.T.A. Hoffmann, à laquelle Sandemose adhère ici, efface à peu près totalement la possibilité d'un idéal humaniste de type classique.

80 John Torson, comme les autres personnages de Sandemose, est un héros sans avenir. Il reste inéluctablement enchaîné à son passé. Le souvenir participe au rôle que joue l'environnement présent dans le drame de John Torson. Poussé par son sentiment de culpabilité, il en devient un personnage à la dimension strindbergienne, même si Sandemose le décrit dans une perspective plus didactique que ne le fait Strindberg. Agissant en somnambule, il tente après coup de jeter un regard lucide sur ses actes.

81 Det svundne er en drøm montre parfaitement comment le temps devient une instance morale, au sens où l'entendait déjà Kierkegaard, une dimension permanente de l'œuvre romanesque de Sandemose. Il n'y a pas de pur présent, donc pas de narration linéaire des événements.

82 À Kjørkelvik Sandemose vit, enfin, des années de tranquillité et de relatif bonheur. Lui qui avait vécu en bohème à Oslo, entouré de sa petite cour au Café du Théâtre, buvant et dissertant copieusement, retrouve enfin un enracinement, réussit à s'accoutumer à son ermitage grâce à sa femme. Mais les contacts avec le monde et l'activité créatrice 
restent intenses. Sandemose intervient dans les polémiques politiques, s'élève contre l'antisémitisme et le réarmement. Il édite la revue Årstidene, dont il est le seul rédacteur, avec des essais et des articles sur des sujets les plus variés, surtout des sujets d'actualité. La revue paraîtra jusqu'en 1955.

À cette époque son champ de la souffrance humaine s'élargit bien au-delà de l'orgueil blessé à vif. Son fils, auquel il avait donné le prénom d'Espen, est atteint d'une maladie incurable et meurt à l'âge de dix ans.

Malgré son chagrin, Sandemose a le temps et le courage de mûrir, dans sa retraite de Kjørkelvik, un projet dont il avait senti jusqu'à présent tout l'inachevé. Car l'autre Espen, celui de Jante, est encore là et continue à le défier.

Et comme un peintre qui fait patiemment des esquisses avant d'en arriver à peindre son chef-d'œuvre, Sandemose reprend cette histoire, son histoire à lui, et refait en 1955 une version remaniée et élargie de En flyktning krysser sitt spor.

En 1944 Sandemose se remarie avec Eva Borgen, une femme née dans la grande bourgeoisie norvégienne. Cela donne une nouvelle assise à la vie de l'auteur et lui permet, de retour en Norvège après la fin de la guerre, d'acheter une ferme à Kjørkelvik, sorte d'ermitage à 250 kilomètres au sud-ouest d'Oslo.

\section{De profundis clamavi}

87 Un fugitif qui revient sur ses pas est incontestablement au centre de l'œuvre de Sandemose, comme un condensé de ses thèmes et de ses moyens d'expression.

Le caractère subjectif de la version de 1933 de ce roman est encore accru. Mais la perspective a changé, les choses se sont décantées. "Déjà en écrivant la première version du livre, nous confie Sandemose, elle m'est apparue comme une page d'archéologie... De nouvelles couches se sont ensuite superposées... Dès la publication du livre, la matière a repris une nouvelle vigueur, et je remarquais que j'étais loin d'en être arrivé à bout... Les parties qui se sont ajoutées entre 1933 et 1955 se reconnaissent à leur ton relativement conciliant. La lutte contre le conformisme y a pris un caractère moins monomane, plus réfléchi ${ }^{10}$. Il s'agira, en 1955, d'un ouvrage où confessions publiques et essai sociologique forment un amalgame inextricable.

89 Le roman, qui frappe par sa forme non conventionnelle, chaotique presque, par l'apparente absence de toute logique et de toute chronologie, par son mélange de récits, de souvenirs, de réflexions et d'explosions soudaines, n'est pas d'une lecture facile. Il est à peu près impossible de le raconter. L'auteur le reconnaît sans ambages : « Au lieu d'agencer mon esprit vagabond et d'inventer des personnages, comme le font les bons auteurs, j'ai laissé les diables qui logent au fond de moi-même - et les anges, car il y en a aussi - raconter tout le temps leur propre histoire, c'est-à-dire la mienne $»^{11}$. On ne saurait indiquer plus clairement qu'il s'agit du cri de détresse d'une âme qui aspire à la libération. "Dans mon cœur, ajoute-t-il, il y a longtemps que j'ai donné au livre un autre nom, après les conciliabules nocturnes que nous avons eus, avec tous mes anges et tous mes démons : "Des profondeurs nous crions vers toi, Seigneur" $»^{12}$.

Sandemose, qui ose enfin parler à cœur ouvert, qui dit lui-même dans la préface qu'il a eu l'impression d'avoir fait sauter un barrage qui le maintenait à un état de pseudoémancipation à l'ombre de son enfance et de Jante, entraîne son lecteur à travers toutes les fluctuations fougeuses de son tempérament, tour à tour tendre et violent, 
mélancolique et enjoué. Au tribunal de l'Histoire il a juré de dire la vérité, toute la vérité sur les causes profondes de ce meurtre par procuration que fut le crime d'Espen Arnakke à Misery Harbor.

91 Le parallélisme entre l'auteur et son personnage est évident. Espen a trente-quatre ans au moment où il rédige sa confession. Dans un long monologue marathon Aksel-Espen Sandemose Arnakke ${ }^{13}$ nous ouvre les dédales de sa psychologie profonde, nous raconte les méandres de sa lutte obstinée contre la dictature des préjugés et la société de Jante. Sa ville natale de Nykøbing Mors en a fourni, bien sûr, le cadre extérieur. La préhistoire du livre, nous la connaissons. Elle est racontée dans En sjømann går iland, et elle reste présente comme toile de fond de la première à la dernière page du livre. Bien plus, elle en est l'aiguillon qui transforme les investigations sur les raisons du crime en un gigantesque $\mathrm{j}$ 'accuse.

93 C'est en fonction de l'échelle des valeurs qu'on lui avait inculquée à Jante qu'Espen s'était approché d'Eva à Misery Harbor. Le chemin de la virilité et du bonheur passait par les femmes, elles étaient la voie. Eva devient pour Espen un symbole de puissance et de prestige social, un moyen pour atteindre l'idéal de virilité que John Wakefieldre présente justement sans complexes et sans retenue. Peu d'autres traits individuels sont soulignés chez ces deux personnages-symboles. John accueille Espen avec une sorte de supériorité et de bienveillance paternelle, mais les relations entre les deux hommes n'en demeurent pas moins ambigus dès le départ. «Le grand John acceptait volontiers qu'Espen devienne son ami, car il ressemblait aux filles $»^{14}$. Le face-à-face du chanceux et du guignard se double ici d'une composante homosexuelle ${ }^{15}$, comme chez Prästen et Gullhästen. Être accepté par le grand John est indispensable à Espen pour pouvoir affirmer sa propre virilité.

Mais après son crime il n'a plus besoin de porter le masque de la puissance virile. Son état psychologique, son monde intérieur, enfin, se débloquent et d'autres valeurs apparaissent. Le fugitif, qui fuit le châtiment d'un crime impuni, qui fuit la société de Jante et la personnalité qu'elle lui a façonnée comme un habit trop étriqué, doit revenir sur ses pas, montrer en pleine lumière son Moi humilié, refoulé, qui surgit dans ses rêves. Il doit avoir le courage de considérer en face sa "pitoyable histoire " faite de lâcheté et de soumission à la dictature des préjugés.

L'auteur nous avertit dès le début que dans son récit il ne sera question que de « choses ordinaires et banales ». Le point de départ de son enquête psychanalytique, il faut le souligner encore, c'est le meurtre de Misery Harbor. Pourquoi les ricanements de John Wakefield déclenchent-ils en lui la réaction meurtrière?

Tout ramène Espen à son passé, parce que les conflits sont restés latents. Il faut chercher des explications, une vérité-excuse ou des circonstances atténuantes dans les antécédents du meurtrier, se dit Sandemose. Défendre l'assassin Espen, c'est accuser la société qui l'a formé. La formule a fait son chemin depuis. Mais pour Sandemose elle ne représente pas un simple élément d'une dialectique révolutionnaire, c'est plutôt la porte ouverte à sa propre libération - « Maintenant je peux tout dire ».

L'enfance d'Espen représentait un paradis à peine entrevu. Il le perdra tôt, le jour où Rose, sa petite compagne de jeu, le repousse d'un air de mépris en lui disant: «Tu es laid !... tu n'iras jamais chez le Bon Dieu ». Espen se voit donc exclu, déjà, son malheur, comme tout malheur, a des racines métaphysiques. «Et je me sentis vraiment bête et laid... Le péché avait fait son entrée dans le monde ». 

semblent pas faire partie de Jante, ils sont comme un havre de paix dans cet océan de haine et de mépris que représente le monde des adultes. Il n'y a pas de conflits ouverts avec eux. Le père est bon et doux, la mère discrète et taciturne.

À la naissance de sa sœur Agnès, Espen se sent pris d'un sentiment de frustration. Sa jalousie l'oblige à sa première fuite, il s'éloigne de sa mère et reporte son affection sur des objets, comme la "pierre sacrée » dans le mur de la ferme d'Adarnsen, une pierre qui rayonne une telle chaleur pour lui.

0 Devant son père, Espen se sent comme le fils prodigue qui a fui la maison. Bien que le père lui interdise d'aller dans la grange d'Adamsen, il ne parle jamais d'affrontements. Cette grange est au centre de son enfance. Les jeunes s'y libèrent des interdits en goûtant au fruit défendu. Elle devient le symbole des tabous sexuels et de l'autorité paternelle qui l'en exclut.

1 Dans ses visions futures et dans ses rêves il la verra se consumer dans les flammes c'est la tentative du jeune garçon de quatre ans, du jeune Aksel, qui en fin se réalise. On veut l'exclure de la grange, mais il sait qu'il pourra y apprendre à devenir aussi grand que le père. Car c'est cela qui importe. Alors il pourra conquérir l'amitié, la considération des autres, les femmes.

2 Sa sœur Agnès, en compagnie de laquelle il se ménage une cachette dans la grange, sera le premier symbole de sa volonté de conquérir les femmes, mais aussi de son impuissance à y parvenir. Des désirs incestueux l'uniront longtemps à elle, et c'est précisément dans les chapitres consacrés à la grange d'Adamsen qu'Espen les évoquera le plus intensément.

03 L'âge adulte est essentiellement identifié à la sexualité. Le besoin d'affection et de sécurité du jeune garçon se transforme alors en besoin de puissance. Cette véritable religion d'Espen n'est pas formulée ni articulée en dogmes, et cependant elle est ressentie de façon permanente. Cela n'empêche qu'il ne sera jamais le surhomme qu'il voudrait être, car sa volonté de puissance est paralysée par la peur. Il fuit le nécessaire combat, par peur et par faiblesse. Il se fuit lui-même.

04 Son affectivité refoulée le rejette dans la solitude. La peur des autres l'entraîne dans des actes insensés, se transforme même en panique pathologique, sans que l'ambivalence des sentiments, attirance et rejet, ne s'éteigne jamais. Ils gagnent même en intensité. Plus grande était l'admiration, et plus grande deviendra la haine, une haine qui n'éclate jamais au grand jour, mais qui traverse tout le livre en sourdine. Il ne s'agit pas d'une philosophie chez Espen, mais d'une attitude fondamentale de déraciné dans la vie. Fuir les contraintes, fuir Jante !

Nykøbing Mors, le modèle de Jante, était au début du $\mathrm{xx}^{\mathrm{e}}$ siècle une petite bourgade industrielle de 6000 habitants, avec une population ouvrière assez homogène. Il y existait une certaine vie associative. La tempérance et le socialisme y étaient les grandes questions dont l'on débattait. Traditionnellement on y distinguait les ouvriers et les " gens bien ». Le jeune Espen avait tendance à ranger parmi ces derniers tous les grands. Ceux de sa famille, les oncles et tantes, dont il parle en phrases haineuses pour les rendre ridicules, ceux qu'il est obligé de fréquenter et qui le dressent, les petits patrons, la maîtresse d'école dont le seul argument est le fouet. Ils sont agressifs et bêtes, durs envers ceux qu'ils dominent, critiquent les autres, ont toujours raison et se drapent dans leur dignité morale. C'est le jeu de la vie à Jante. 
106 La description qu'Espen nous en donne tout au long du livre est très orientée, obsessionnelle, sans volonté réelle de comprendre. C'est un jeu de tyrans, et Jante en est peuplée, du bourgeois jusqu'à l'ouvrier. Cette ville devient ainsi dans l'esprit de l'auteur une véritable abstraction, synonyme de toutes les formes d'oppression. L'individu y est opprimé au nom d'un code non écrit, la « loi de Jante ».

Il s'agit d'une série d'interdits qui composent cette loi, dont l'énumération monotone souligne le caractère stupide et grotesque. Sandemose se fera un titre de gloire d'avoir rédigé cette loi, qui deviendra un véritable concept dans la littérature scandinave. Tous ces commandements, dont la fausse solennité singe intentionnellement la loi de Moïse, concernent l'attitude de l'individu envers la collectivité, et non pas ses actes. "Tu ne dois pas croire que tu sois quelque chose... Tu ne dois pas... tu ne dois pas... ». Grâce à ces commandements «Jante terrorise Jante ». L'homme n'y existe qu'en fonction des autres, il n'est jamais un individu autonome. La valeur suprême, c'est une collectivité sans communauté, à laquelle il faut des boucs émissaires. Espen affirme que chacun y vit sous la surveillance de chacun, tirant sa respectabilité de l'abaissement de l'autre. Le chanceux que chacun voudrait être doit d'abord se fabriquer son minable, en faisant surgir en l'autre le sentiment qu'il ne vaut pas grand-chose.

108 L'enfer, c'est les autres - la formule de Sartre s'applique déjà à Jante et pourrait en résumer toute la loi. Espen se heurte d'autant plus à elle que son intelligence lui permet d'en percer le caractère pervers.

109 L'atmosphère de suspicion permanente et de délation qui règne à Jante, depuis la famille jusqu'à l'usine, crée une névrose spirituelle généralisée. La terreur morale exige le péché, ainsi l'on pourra pardonner et intensifier sa prise sur une victime qui, comme chez Kafka, se bat avec ses remords et son sentiment de culpabilité.

110 Jante, avec sa loi morale, montre bien avant Hitler comment le fascisme s'empare de l'intérieur d'une cité ${ }^{16}$ et la transforme en véritable camp de concentration. Mais ce n'est pas un émule de Hitler qui organise la terreur à Jante, ce sont ses propres habitants, par la fausse démocratie du conformisme et par la perversion de l'idéal d'égalité. Sandemose décortique dans son livre, de façon froide et lucide, la structure interne de la dictature collective. C'est la masse anonyme qu'il rend responsable de cette dictature sans dictateur. Elle est la première à en souffrir - Jante terrorise Jante mais Sandemose ne peut la défendre, car elle est irrémédiablement livrée à la bêtise. La Bêtise, voilà l'ennemi, l'infâme qu'il faut écraser. Si elle a des accents voltairiens, la révolte de Sandemose contre la dictature fascisante du nivellement social rappelle aussi la rébellion d'Ibsen contre la « majorité compacte ».

111 Les membres de la classe ouvrière sont loin d'être les derniers à contribuer à l'oppression générale, à l'ordre moral. En dénonçant le conformisme, Sandemose ne se fait pas pour autant le thuriféraire de la classe ouvrière. Bien au contraire.

$112 \mathrm{Vu}$ sous l'angle de la psychologie, il n'y a pas de différence essentielle entre l'ouvrier et le bourgeois. Jante est une collectivité ouvrière où la notion de solidarité est absente.

113 Le processus de socialisation n'intéresse pas cet individualiste qu'est Sandemose. Son expérience de la vie l'a même amené à se méfier de plus en plus des doctrines de rédemption sociale. Sa révolte à lui prend les allures d'un combat singulier, à la Strindberg, ce qui fait du révolté un misanthrope, un négateur. Il ne donne pas, à l'opposé de Nexø, dans l'ouvriérisme, cette idéalisation un peu naïve du monde du travail. En flyktning krysser silt spor peut même être considéré, dans une large mesure, 
comme son antidote ${ }^{17}$. Donner une idée fausse et lénifiante de la classe ouvrière serait rétrograde aux yeux de Sandemose, et relèverait du préjugé social.

Les inhibitions dans le domaine sexuel, la religion du travail et l'habitude de la discipline chez les ouvriers en font une classe sans esprit et sans idées.

Sandemose décrit une collectivité ouvrière qu'il a bien connue et ne prétend pas peindre dans l'universel. Mais il constate que l'instinct de conservation y pousse chaque individu à réaliser sur le plan horizontal les modèles de relations qu'il voit et qu'il vit sur le plan vertical. Le sens de la hiérarchie plonge ses racines jusque dans l'inconscient, de sorte que les membres de la classe ouvrière cherchent instinctivement à établir entre eux des relations de type vertical comme elles existent déjà dans la société de classes.

Chez les jeunes ces tentatives hiérarchisantes sont particulièrement intenses et violentes sur le plan sexuel. À tel point que le jeune Espen, à l'âge de la puberté, se masturbe quotidiennement pour se prouver qu'il va être un homme. Il n'y a aucune connotation sensuelle ou voluptueuse dans ce fait. Même la masturbation devient un acte social.

La concurrence érotique qui détermine la vie aboutit forcément à une situation triangulaire. Le triangle symbolise la lutte pour la domination. Malgré ses efforts, Sandemose ne se détachera jamais complètement de cette conception. Et Espen ne sortira de cette constellation psychologique que par le meurtre à Misery Harbor.

118 Faut-il alors considérer cet acte comme une fatalité ? Sandemose ne fait pas sienne, malgré les apparences, une conception purement déterministe de l'homme. Bien que la doctrine freudienne ait donné des arguments nouveaux à la théorie de l'influence du milieu, celle-ci lui paraît bien trop mécanique, ne laissant guère de place aux impondérables ni à l'activité psychique propre de l'individu.

119 Voulant saisir in vivo cette activité psychique, Sandemose en arrive forcément à donner à son livre la forme que nous lui connaissons, une forme chaotique, achronologique, qui apparaît si déroutante à la première approche.

120 Le récit couvre une période de trente deux ans environ, l'accent principal étant mis sur la période de la puberté. Ce n'est pas une rétrospective rigoureuse. Il ne s'agit pas non plus de "mémoires", mais de souvenirs, d'expériences vécues qui se bousculent, s'enchaînent, bouillonnent dans le cadre fictif du meurtre de Misery Harbor. Les scènes se passent pêle-mêle à Jante, à Terre-Neuve, au Canada, à Copenhague, en Norvège ou en mer. On change constamment de perspective et de plan chronologique.

Les changements d'optique et d'époque s'opèrent sans que le lecteur ne soit averti ni préparé, sans commentaires, parfois à l'intérieur même d'un chapitre. Grâce à cette technique narrative, proche de celle de Strindberg dans Le Fils de la servante, l'auteur peut revenir sans cesse sur des scènes-clefs, les sonder pour en comprendre le sens profond.

122 Dans ce monologue intérieur, qui procède par association d'idées méthode dont se sert la psychanalyse - la seule cohérence est celle de l'inconscient. Le passé et le présent se fondent dans des visions parfois hallucinatoires. Le passé est toujours présent, il existe dans la conscience d'Espen, et sans la confession libératrice le futur n'en serait que l'ombre, avec la même problématique, les mêmes protagonistes.

La méthode a chronologique du récit, qui a été également employée par Faulkner, fait sentir davantage le poids d'une destinée sombre. Il s'en dégage un sentiment 
d'immobilisme, de pesanteur qui ne provient pas du récit, mais de la vie elle-même. Cette forme a sans doute été ressentie comme nécessaire par Sandemose, car un récit chronologique n'aurait pu être que fade, avec des redites permanentes de «choses ordinaires et banales ». La forme du livre est l'expression directe de l'intemporalité à laquelle se sont hissé les événements relatés. Le héros, caractère blessé, déchiré, vit et revit ainsi sa vie sous le regard de "l'autre». C'est un huis-clos dramatique, d'un orgueil pesant. Espenlefugitif, le meurtrier, évolue dans un monde fermé, avec sa sensibilité à vif, à la recherche d'une ouverture, d'un rayon de soleil.

\section{Erlingvik, ou le pays qui n'est pas}

Après cette gigantesque analyse, Aksel Sandemose peut tourner la page, il peut enfin surmonter sa haine, connaître une période plus apaisée et faire entendre l'appel à la réconciliation. L'outsider persécuté par lui-même, l'errant inquiet s'achemine vers une relative sérénité dans son refuge de Kjørkelvik, sous l'influence notamment de sa femme Eva Bargen.

Celle-ci l'aide activement dans son travail préparatoire à un nouveau roman, Varulven (Le loup-garou $)^{18}$, car elle a été mêlée aux événements qu'il relatera.

La vie vécue continue, en effet, à fournir la trame du récit, bien que Sandemose prenne $\mathrm{du}$ recul et de la hauteur, et domine davantage sa subjectivité. Le temps des règlements de compte est terminé. Dans ce roman, le Mal n'est plus affronté directement dans ses œuvres vives, il atteint les dimensions du mythe.

Le loup-garou, c'est le symbole des puissances maléfiques que Sandemose a cherché à combattre, l'image de l'emprise démoniaque sur l'homme. C'est tout à la fois l'envie qui ronge le guignard, l'intolérance et la soif de puissance qui habitent le chanceux, c'est la haine de l'autre et la jalousie qui fait de l'homme son propre bourreau. L'appétit de ce loup-garou est insatiable, il trouve des proies faciles en rôdant dans les ténèbres de l'ignorance et du fanatisme. La période de l'occupation nazie de la Norvège, où se situent beaucoup de scènes du roman - on ne peut guère parler d'action proprement dite - est particulièrement propice aux agissements de cet être de terreur. Les légions de la Bêtise, ce venin mortel des civilisations, une fois de plus se déchaînent et deviennent les meilleurs auxiliaires de la dictature. Comme cela fut déjà le cas des autres œuvres romanesques de Sandemose, il est à peu près impossible de résumer le contenu de ce livre. Le caractère heurté de la composition se confirme et montre que l'auteur a bien trouvé là la forme d'expression la plus conforme à son génie.

Il s'agit de tranches de souvenirs qui se suivent et s'interpénètrent, comme dans En flyktning krysser sitt spor. Des passages qui pourraient relever du roman policier voisinent avec des considérations sur l'histoire de la Norvège, avec des épisodes à horsd'œuvre, y compris des recettes de cuisiner apportées sur une page entière.

En fait, Sandemose « digère » dans ce livre - parfois il le fait mal - ses impressions et souvenirs de guerre. Avec un certain détachement, certes, de même qu'il analyse la résistance norvégienne contre le nazisme avec plus d'objectivité que dans Det svundne er en drøm. Il y a les actes de résistance, et il y a les mobiles. Et au vu de ces derniers, tout idéalisme se dissipe comme une brume au soleil. Pour le jardinier Tor Andersen, une des nombreuses incarnations du loup-garou, la résistance n'était qu'une vengeance privée, un compte à régler avec des soldats allemands qui avaient osé se moquer de lui. 
Pour guider le lecteur il y a les personnages centraux qui servent d'axe au récit. Erling Vik est un écrivain vieillissant, né le même jour et la même année que Sandemose. Partageant son temps entre les cafés de la capitale et son refuge à la campagne, il essaie de mettre de l'ordre dans sa vie et dans ses souvenirs. Mais il est plus soucieux encore de se ménager et sa liberté et sa cachette à boissons.

131 À la campagne il retrouve sa maîtresse, Felicia Venhaug, la femme de son ami Jan qui est tout à fait complice de cette liaison. Les rapports de force à l'intérieur du fameux triangle se sont donc déplacés. Felicia, qui a traversé les années de guerre à côté d'Erling, est décrite comme une des incarnations de la séductrice, de l'Eve qui prend plaisir à aguicher et à tourmenter les hommes. C'est ainsi, par exemple, qu'elle s'enferme dans la serre de la propriété pour y dévoiler ses charmes en toute liberté, sachant que le pauvre jardinier Tor Andersen s'est ménagé des trous dans la paroi pour la dévorer des yeux. Elle est d'un orgueil profond qui la pousse à manifester son mépris à tous les faibles, portant ainsi un des multiples masques sous lesquels se cache le loupgarou.

132 Sandemose continue volontiers à accabler la femme, à en faire le symbole et la cause du mal. Felicia Venhaug est une imagevivante de l'éternel féminin au yeux de Sandemose, une illustration de sa conception de la femme, conception machiste dont il ne veut $\mathrm{ni}$ ne peut se défendre. Demeurant sans rémission l'Eve, la cause de la chute, Felicia disparaît d'ailleurs mystérieusement un soir de février, alors que la neige se met à tomber, comme happée par un autre monde.

L'histoire de Gulna revient à l'appui de cette vision de la femme. Cette jeune fille, dont le nom était devenu dans Vi pynter oss med horn celui de la déesse de l'inaccessible, a été la première amourette du jeune Erling. Leur idylle ayant été découverte par ses parents, ceux-ci la battent jusqu'au sang pour l'obliger à avouer des «fautes » qu'elle n'a même pas eu le temps de commettre. Soumise à un véritable lavage de cerveau, qui la traumatise et la culpabilise, elle se voit irrémédiablement enfermée dans un univers de faute morale, victime d'une pédagogie tyrannique dont Sandemose affirme - il en a toujours été convaincu - qu'elle cherche à faire des enfants des invalides de l'esprit et des nains intellectuels. Lorsque trente ans plus tard, arrivée à l'âge adulte, Gulnare rencontre de nouveau Erling, elle l'insulte publiquement et maudit son "séducteur ", en véritable mégère de la vertu outragée. La victime est devenue à son tour bourreau, le loup-garou a pris possession d'elle.

134 Assez curieusement, il y a dans le livre un personnage féminin, Gustava, la vieille voisine avec laquelle Erling a pris l'habitude de venir bavarder pour se détendre, qui semble échapper à la fatalité qui fait de la femme un des supports par excellence de la tyrannie collective.

135 Vivant en marge, elle incarne le droit de l'individu à dire non à la société et à son faux évangile de la solidarité. Comme elle récuse tous les prophètes, les sauveurs et les dogmes, elle ne s'occupe pas non plus des autres et respecte la liberté de chacun. Citoyenne d'un monde autre, qui n'est pas, d'un anti-Jante, elle représente en quelque sorte l'idéal moral de Sandemose. Son exemple illustre cependant le risque que court chaque être, dans son individualisme sans concessions, de se voir enfermé dans un univers rétréci, dans une solitude existentielle irrémédiable. 

Holbaek, qu'il emmène avec lui dans sa semi-retraite à la campagne. Et en 1964 il intervient de façon vigoureuse - et intempestive aux yeux de certains - dans une affaire qui secoue la ville de Bergen, l'affaire Schnitler. Il s'agit d'un jeune criminel qui a violé et assassiné une jeune fille. Sandemose pense que cette affaire interpelle et la Norvège tout entière et la société moderne. Il prend la défense du jeune Schnitler contre les attaques de la presse à sensation et affirme que son cas relève de l'hôpital psychiatrique et non pas des tribunaux.

Cette prise de position montre que les ombres de Prasten et d'Espen Arnakke rôdent encore et peuplent les songes de Sandemose, que le drame de son moi, cas exemplaire et significatif, continue à travers les autres. L'auteur reste au centre de son œuvre, et son attitude constante de rédacteur de journal intime qui se confesse et présente ses comptes, se poursuit même à travers la vie et les avatars d'autrui. 
Sandemose se sera méfié jusqu'au bout de la fiction. Ce qu'il appelle son «honnêteté » l'empêche de se servir de son imagination, et les œuvres où il veut garder une distance épique, s'abriter si possible derrière une fiction, sont sans doute les moins réussies. Sa vie a donné à l'écrivain suffisamment de matière - et de fil à retordre - pour qu'il n'ait pas beaucoup besoin de recourir à la fiction, à l'intrigue née de son imagination.et aspect de son génie n'est pas sans rappeler une certaine parenté avec des auteurs comme Hjalmar Söderberg.

La méthode d'écriture de Sandemose reste jusqu'au bout une méthode didactique. C'est un trait caractéristique qui traverse son œuvre et qui explique la forme si peu conventionnelle de ses romans. Il sait habilement doser l'agressivité du monologue du moraliste et l'intimité de la lettre privée, Car le lecteur doit être mis dans la confidence, C'est pour cela aussi qu'il écrit. L'art de l'allusion et du récit symbolique, qu'il manie souvent avec virtuosité, lui permet d'étaler avec la pudeur voulue son univers intérieur, ses « suicides manqués » comme il appelle ses œuvres ${ }^{20}$.

Aksel Sandemose est bien typiquement scandinave, à la fois par le monde qu'il évoque et dans lequel il se débat, et par la façon dont il relate ses luttes et ses angoisses, sa mauvaise conscience, Comme le fait la mythologie nordique, il partageait volontiers les hommes en fonction de leur chance plutôt que de leur morale, Il n'y a ni bons ni mauvais, il y a des gagnants et des perdants. Ces sont des types de personnages que l'on retrouve dans la conscience des peuples du Nord tout au long des siècles, Le chanceux incarne l'orgueil et l'arrogance, vertus majeures dans la mentalité des anciens Scandinaves, qui déterminent le rang et le destin de l'homme. Le perdant, c'est le solitaire apatride, le banni arraché à la sécurité - la fausse sécurité, selon Sandemose de la tribu, qui ne trouvera plus de repos, même pas dans la mort.

Entre les deux pôles se forge le mythe chez Aksel Sandemose. Dans sa vieillesse encore il écrira qu'il se sentait un poète maudit, né sous une mauvaise étoile.

149 Scandinave de par ses origines, Sandemose le reste jusqu'au bout, car il n'a pas été touché par la grâce du message gréco latin ni par celui du christianisme. Il les récuse d'ailleurs violemment. L'idéal du Beau et du Bien lui demeure étranger. Son monde est un monde rude qui exclut la pitié pour les vaincus de la vie. L'exaltation de la virilité, une virilité vécue comme une jouissance suprême ou comme un cauchemar de l'inaccessible, le confirme dans un individualisme proche de l'anarchiste. Le sang et le sexe y sont les catalyseurs troubles de la violence, et la femme n'est que celle qui vient déranger la sérénité et la hiérarchie des mâles.

150 Cette image négative de la femme va de pair avec l'absence étonnante de toute poésie dans la vie et dans l'œuvre de Sandemose. Celle-ci est tout au plus ressentie comme une intrusion, une distraction, un luxe qui n'est pas de saison. Dans ce monde rude où l'alcool, ce bon vieil alcool qui était déjà le symbole de la révolte du jeune Espen contre Jante, sert de refuge et d'argument suprême, les affrontements forment la trame de la réalité quotidienne.

151 La lutte contre l'hypocrisie et la lutte contre «l'autre » se confondent chez Sandemose en une gigantesque lutte contre l'Ange, contre l'intrusion du surmoi, un surmoi trop rigide qui fait de l'homme son propre persécuteur. Et après avoir lutté contre l'Ange, dit la Genèse, Jacob boitait de la hanche. Une infirmité qui restera aux yeux d'Israël le signe visible et symbolique de la lutte. 
Sandemose lui aussi a porté longtemps la marque de sa lutte contre l'Ange, comme une plaie ouverte. L'aiguillon de la haine, puisqu'il faut et puisqu'il veut appeler les choses par leur nom, l'avait stimulé dans la lutte. Mais la haine avait pour lui une valeur positive d'épuration.

153 Sa plaie s'était-elle complètement refermée ce jour de printemps 1965 où il put enfin retourner à Jante, où la ville de Nykøbing Mors reçut son fils prodigue en invité d'honneur?

Peu après, le six août de la même année, Sandemose mourut d'une attaque d'hémiplégie.

Le périple de ce marin au long cours, de celui que Vesaas appellera un "oiseau migrateur » et Eyvind Johnson « un type bien » - en bra människa - s'achève, et il ferme ses paupières sur son visage buriné, sonnez d'aigle qui faisait penser à un sabre, et ses longues rides qui encadraient comme des cicatrices une bouche un peu trop amère. Il avait écrit son message pessimiste non pas sur du sable, mais sur les vagues d'un océan sans havre et sans sécurité. "La vérité est écrite sur de l'eau, affirme-t-il dans la présentation du livre de sa vie, et ce que l'on appelle justice est le cauchemar de quelques braves gens $»^{21}$.

\section{NOTES}

1. Sigurd Hoel était critique littéraire chez Gyldendal et écrivain. Il est parmi ceux qui ont le plus stimulé Sandemose et contribué à le faire connaître.

2. Bien que ne suivant nullement ses traces, Sandemose se souviendra longtemps de ce poète penché avec mélancolie sur les obscurs sentiers du Moi.

3. Les écrits de Freud ont été introduits au Danemark par Otto Gelsted. Des jeunes poètes comme Tom Kristensen se sont avidement emparés du nouvel évangile. Mais la psychanalyse chez Kristensen présentera des allures essentiellement négatives, le Moi profond étant ressenti comme une charge pesante dont il faut se libérer. En Norvège ce fut Sigurd Hoel qui, le premier, utilisa la psychanalyse vers les années 1920 comme instrument d'écriture romanesque.

4. Sandemose en gardera une grande rancune. Il partira en guerre contre le lobby des éditeurs. Dans une conférence prononcée en avril 1934 il attaqua violemment Harald Grieg, le directeur de Gyldendal, et la tutelle économique insupportable à laquelle étaient soumis les écrivains. L'Association des écrivains norvégiens prit cependant dans cette affaire fait et cause contre Sandemose, considéré encore comme un étranger.

5. Le titre même est déjà une référence à la psychanalyse et à la méthode freudienne de traitement psychanalytique.

6. La revue ne connut que quatre numéros.

7. Titre de la traduction française de Mme Manceron, parue aux Éditions Stocken 1940. Littéralement le titre norvégien signifie : Nous nous parons de cornes.

8. Cet aspect se discerne déjà dans le roman de jeunesse Ungdomssynd.

9. Ulla Alfredsson, Språk, sexualitet, fascism : en studie i Sandemoses roman Vi pynter oss med horn, Göteborg, 1976.

10. Aksel Sandemose, En flyktning krysser sitt spor, p. 18-20. 
11. Id., p. 19.

12. Ibid.

13. Sandemose indique dans sa préface qu'il a emprunté le nom d'Espen à la légende d'Esbern et Aksel, les deux fils jumeaux d'Asser Righ, et que le nom d'Arnakke est celui d'un petit promontoire près de sa ville natale où il aimait se rendre dans sa jeunesse. Le nom même révèle donc déjà, et intentionnellement, la parenté profonde entre l'auteur et son personnage.

14. En sjømann går iland, p. 48. Gyldendal Norsk Forlag 1931.

15. Cette interprétation est notamment soulignée par Tryggve Braatøy dans Kjaerlighet og hat, article d'inspiration freudienne publié dans la revue Edda, 1933.

16. Il est probable que la montée du fascisme, dont Sandemose était un adversaire déterminé, lui fait prendre conscience de façon plus directe de cet aspect des choses. Dans la version de 1955 de son livre, en tout cas, son analyse a posteriori est tout à fait pertinente.

17. C'est la thèse de Hans-Jørgen Nielsen dans un article Arbejderforjatteren Sandemose of arbejderismens overvindelse paru dans Vinduet 3/4, 1979.

18. Traduit du norvégien par E. Eydoux.

19. Cark-Eric Nordberg, Sandemose, p. 269, Bonniers, 1968.

20. «Je n'ai jamais publié quelque chose sans en être tourmenté par la suite, car il y a un aspect suicidaire dans tout ce que je fais, et il n'est pas bon d'étaler au grand jour ses suicides manqués » (A. Sandemose, in Årstidene).

21. A. Sandemose, En flyktning krysser sitt spor, p. 22, éd. 1965.

\section{AUTEUR}

\section{GEORGES UEBERSCHLAG}

Université de Lille III 